\title{
Real-time monitoring of drug pharmacokinetics within tumor tissue in live animals
}

Ji-Won Seo ${ }^{\# 1,2}$, Kaiyu Fu Hl, 2, Santiago Correa $^{3}$, Michael Eisenstein $^{1,2}$, Eric A. Appel $^{3 *}$, and H. Tom Soh ${ }^{1,2 *}$

${ }^{1}$ Department of Electrical Engineering, Stanford University, Stanford, CA 94305, USA

${ }^{2}$ Department of Radiology, School of Medicine, Stanford University, Stanford, CA 94305, USA

${ }^{3}$ Department of Materials Science \& Engineering, Stanford University, Stanford, CA 94305, USA.

\#These authors contributed equally to this work.

* Correspondence to eappel@stanford.edu and tsoh@stanford.edu

Keywords: nanoporous electrodes, tumor tissue, pharmacokinetics, microelectrode array, antibiofouling, implantable biomedical device, biosensor 


\begin{abstract}
The efficacy and safety of a chemotherapy regimen fundamentally depends on its pharmacokinetics. This is currently measured based on blood samples, but the abnormal vasculature and physiological heterogeneity of the tumor microenvironment can produce radically different drug pharmacokinetics relative to the systemic circulation. We have developed an implantable microelectrode array sensor that can collect such tissue-based pharmacokinetic data by simultaneously measuring intratumoral pharmacokinetics from multiple sites. We employ gold nanoporous microelectrodes that maintain robust sensor performance even after repeated tissue implantation and extended exposure to the tumor microenvironment. We demonstrate continuous in vivo monitoring of concentrations of the chemotherapy drug doxorubicin at multiple tumor sites in a rodent model, and demonstrate clear differences in pharmacokinetics relative to the circulation that could meaningfully affect drug efficacy and safety. This platform could prove valuable for preclinical in vivo characterization of cancer therapeutics, and may offer a foundation for future clinical applications.
\end{abstract}




\section{Introduction}

Inter-individual differences in pharmacokinetics (PK) can profoundly affect the efficacy of drug treatment, particularly in the context of chemotherapy for cancer. ${ }^{1,2}$ This variability makes it challenging to identify the appropriate therapeutic window for a given drug regimen. Underdosing reduces the likelihood of successful treatment, whereas overdosing can inflict severe damage on the kidney, liver, heart, and other organs. ${ }^{3-7}$ Currently, such PK data are typically collected via blood-based measurements of circulating drug concentrations, and a number of groups have even demonstrated the feasibility of real-time drug monitoring through the use of miniaturized implantable sensors. ${ }^{8-11}$ However, such circulation-based measurements do not adequately reflect drug absorption within the tumor tissue itself. ${ }^{12-16}$ This is because the microenvironment within tumors is complex, with unpredictable vascular permeability, heterogeneous and high interstitial fluid pressure, high cell density, and disorganized lymphatic drainage. ${ }^{17-19}$ These factors can collectively impede the continuous and homogenous penetration of drugs from plasma into the tumor tissue, resulting in notable differences in drug concentration between the plasma and different regions of the tumor. As such, measurements of drug concentrations in plasma can yield inaccurate assessments of PK, resulting in low therapeutic efficacy.

Currently, the only way to obtain tumor-specific PK measurements is through tissue specimens collected via needle-based biopsies. However, it is difficult to extrapolate overall drug penetration in the heterogeneous tumor tissue environment from a single sampling site, and this in turn leads to inaccurate PK assessment. Multiple biopsies would offer a more complete picture of tumor PK, but it is impractical to perform this invasive procedure repeatedly, because it is costly, time consuming and carries the risk of severe side effects-including tumor seeding. ${ }^{20-23}$ Even in animal models, multiple biopsies of tumors are technically challenging, and so researchers typically carry out PK studies by collecting samples from multiple animals sacrificed at different time-points, producing averaged population data that do not accurately capture intra-tumor variability from individual animals. ${ }^{24}$ Furthermore, these experiments are being performed ex vivo, and may not accurately capture the physiological behavior of a tumor within its in vivo milieu. More generally, such analyses are challenging to perform for a variety of reasons - for instance, the probe needs to withstand insertion into solid tissue with minimal sensor damage, and must be sufficiently resistant to biofouling to enable robust measurement over extended periods of time. Accordingly, there remains an unmet need for analytical tools that are capable of efficiently collecting accurate PK data from multiple tumor sites simultaneously. 
Here, we demonstrate an electrochemical aptamer-based biosensor that enables robust, real-time, multi-site drug monitoring within tumor tissue in live animals. Our biosensor features a number of technical and design advances that enable it to overcome key limitations that have hindered past efforts to achieve drug monitoring within solid tissues. First, we make use of nanoporous gold microelectrodes that successfully minimize both the effects of fouling from biological matrices and the risk of sensor damage from tissue insertion. Second, each sensor incorporates several such microelectrodes so that we can monitor drug concentrations with a higher signal-to-noise ratio (SNR) at multiple sites within tumor tissue simultaneously. Finally, our sensor is flexible, offering a better match to the physical properties of surrounding tissue and thereby minimizing damage at the site of insertion. As a demonstration, we show that our microelectrode array sensor can monitor concentrations of the chemotherapy drug doxorubicin (DOX) at multiple positions in tumor tissue simultaneously. This enables us to collect in situ tumor-specific PK data that account for tissue heterogeneity within a single animal, revealing patterns of DOX distribution within the tumor tissue that differ starkly from those measured via the systemic circulation. These differences indicate that the latter metrics might prove misleading in the selection of an appropriate drug dose, and highlight the importance of in situ PK monitoring in the context of cancer therapeutics research. Our data indicate that our biosensor platform could offer a simple and robust tool for obtaining more physiologically relevant insights into drug PK and understanding the in vivo behavior of experimental drugs. 


\section{Results and discussion}

\section{Overview and fabrication of the sensor}

Our sensor comprises an array of gold nanoporous microelectrodes integrated into a flexible, polyimide polymer-based probe, which can be implanted directly into tumor tissue in a live mouse (Figure 1A). This sensor is designed to collect temporal drug concentration profiles at multiple sites within tumor tissue simultaneously via multiple microelectrodes in real-time (Figure 1B). Based on the resulting measurements, the PK of the drug can then be assessed in terms of its absorption and elimination phases, where the former refers to the drug's uptake into the tissue from the circulatory system and the latter describes the drug's subsequent clearance from the tissue due to lymphatic drainage and other physiological processes. ${ }^{25,26}$ The mean intratumoral PK of the drug — as calculated based on concentration data obtained at three different microelectrode channels - can then be compared to the systemic PK (Figure 1C).

Detection is achieved by functionalizing the surface of these gold microelectrodes with aptamers for the drug of interest that undergo a conformational change upon binding to their target. For the present work, we used a well-characterized aptamer that can bind DOX - a widely used chemotherapeutic drug. ${ }^{27-31}$ As described in our previous work ${ }^{32}$, the distal end of the aptamer is tagged with a methylene blue (MB) redox reporter; in the presence of the target, the aptamer undergoes a conformational change that increases the rate of electron transfer between the MB reporter and the electrode surface, thereby yielding an increase in current (Figure 1D). We applied square-wave voltammetry (SWV) to determine the signal gain, which is the ratio of current measurements from before and after target addition. Since the aptamer can reversibly bind and release its target, our sensor can continuously measure target concentration and kinetic information in real-time. 
(A)

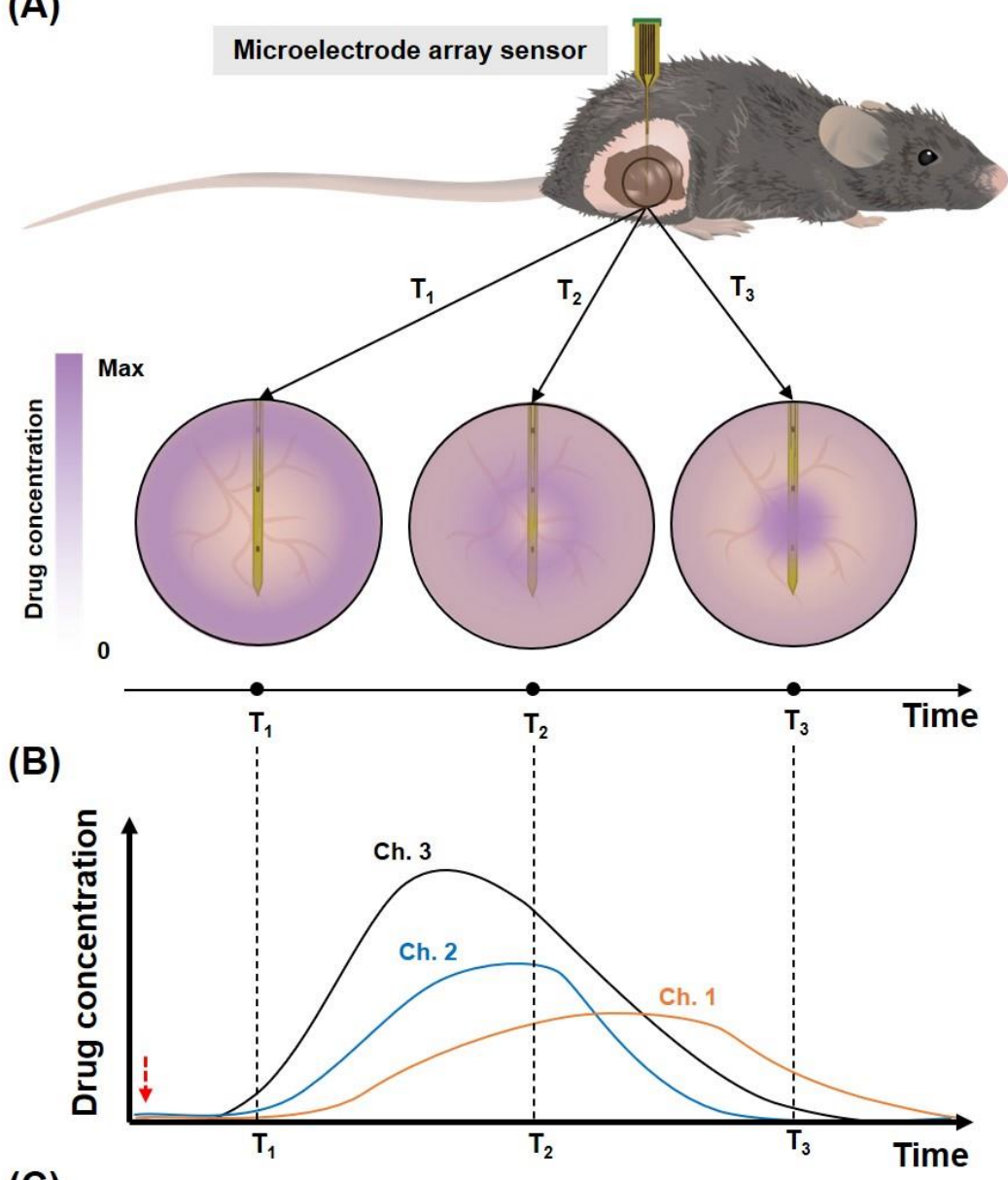

(C)

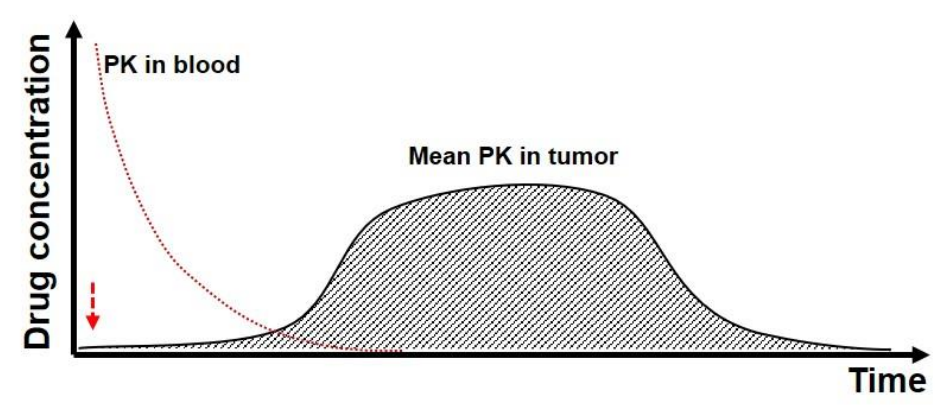

(D)

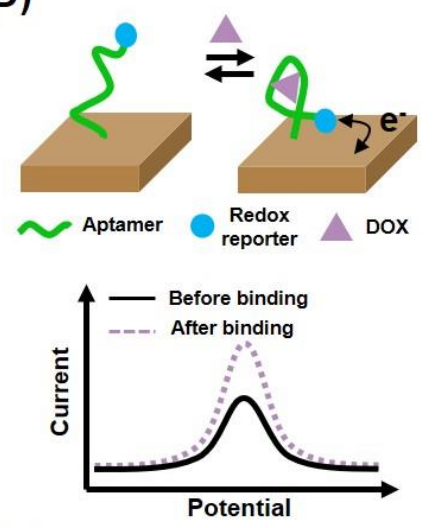

(E)

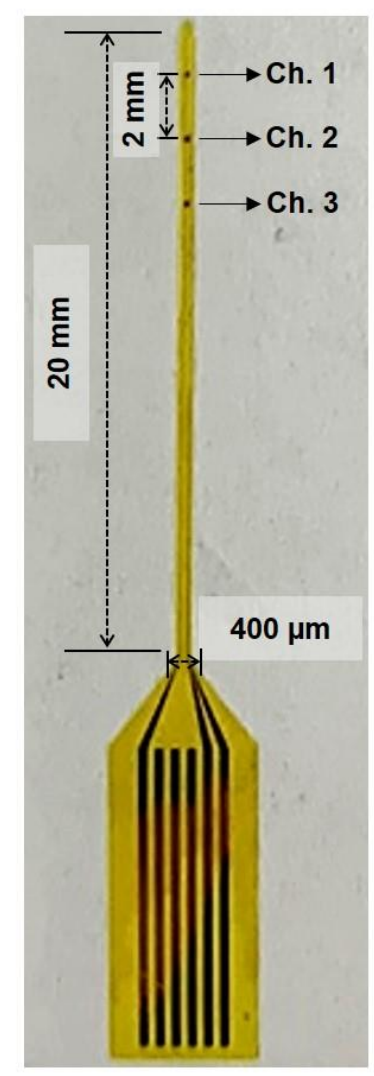

Figure 1. Gold nanoporous microelectrode array-based implantable electrochemical aptamer sensor. (A) Illustration of sensor implantation into tumor tissue in a live mouse (top) and detecting drug concentrations within the tumor at different time-points $\left(\mathrm{T}_{1}, \mathrm{~T}_{2}\right.$, and $\mathrm{T}_{3}$; bottom). (B) Illustration of real-time measurement of different drug concentrations at each microelectrode channel. Dotted lines represent the time-points from (A). (C) Illustration of averaged real-time drug concentrations from the three sensor channels versus drug concentrations from blood. Red dotted arrows in (B) and (C) indicate the time of drug injection. (D) Schematic of the aptamer-based drug detection mechanism. (E) Photo of our sensor.

We recently reported that nanoporous structured electrodes offer greatly improved electrochemical detection sensitivity relative to planar electrodes, with a higher signal-to-noise ratio (SNR) due to reduced charge-screening effects. ${ }^{33}$ This enhanced sensitivity is important in this context, because in vivo experiments intrinsically have high background noise. The use 
of nanoporous microelectrodes should also facilitate long-term monitoring in tumor tissue, because aptamers residing within the nanopores are better protected against both mechanical damage during insertion and biofouling during implantation. ${ }^{34,35}$ Each sensor comprises an array of several such nanoporous gold microelectrodes fabricated onto a flexible polyimide substrate, which forms a 400- $\mu \mathrm{m}$ x 20-mm shank with a thickness of $15 \mu \mathrm{m}$ (Figure 1E and S1). The entire fabrication process is detailed in the Supporting Information (Figure S2). Briefly, an Au:Ag alloy film was deposited onto the polyimide substrate by co-sputtering of $\mathrm{Au}$ and Ag, after which the silver was dissolved in 69\% nitric acid (Figure S3A). To prevent degradation of the polyimide substrate during this process, we added a gold bottom-protective layer before deposition of the Au:Ag alloy (Figure S3B). We then functionalized these microelectrodes with the MB-tagged DOX aptamer. For this work, we used a three-channel array consisting of $100 \times 100 \mu \mathrm{m}^{2}$ microelectrodes positioned with a 2 -mm pitch. This pitch enables the sensor to measure a large area of tumor tissue simultaneously, while the relatively small area of the microelectrodes confers high spatial resolution compared to needle biopsies or metal wire-based sensors. The sensor was connected to a printed circuit board (PCB), which was in turn connected to a commercial potentiostat (Figure S4). The working electrode array in the sensor and a conventional $\mathrm{Ag} / \mathrm{AgCl}$ reference electrode were used together for all measurements. After recording the SWV curves with the potentiostat, we utilized a custom MATLAB script to calculate the DOX concentration.

The mechanical flexibility conferred by our polyimide substrate is another essential feature of our in vivo sensor. It is well known that sensors made of silicon or metal wires lead to a mismatch in mechanical properties between the sensor (stiffness $\sim 1 \mathrm{mN} \cdot \mathrm{m}$ ) and surrounding tissue $(\sim 100 \mathrm{nN} \cdot \mathrm{m})^{36}$, inflicting damage on the tumor tissue. Such tissue damage could lead to inaccurate measurement, prevent long-term monitoring, or cause additional tumor seeding. ${ }^{22}$ Our flexible probe minimizes such risks because its stiffness is sufficiently low ( 270 $\mathrm{nN} \cdot \mathrm{m}$ ) to approach that of tumor tissue. ${ }^{37-39}$

\section{Characterizing sensor performance of our microelectrode array}

We initially tested the performance of our sensors in a series of in vitro experiments. We first confirmed that each microelectrode in our array has equivalent sensitivity. Briefly, we immersed our sensor in 1x saline sodium citrate (SSC) buffer and introduced $10 \mu \mathrm{M}$ DOX after allowing the baseline signal to stabilize for 12 minutes. All three channels showed a similar response, producing an average $\sim 53.9 \%$ signal gain with $1.4 \%$ variance between channels (Figure 2A). At $\mathrm{t}=17 \mathrm{~min}$, we washed the sensor, and the signal returned to baseline within 
minutes, confirming the capacity for continuous, real-time sensing. We next exposed our multichannel sensor to increasing DOX concentrations, and observed a clear and proportional signal gain; our three electrodes exhibited a steady increase in signal gain from $2.9 \%$ at $500 \mathrm{nM}$ to $78.1 \%$ at $30 \mu \mathrm{M}$, with just $3 \%$ variance in signal gain at each concentration across the three electrodes (Figure 2B). We calculated the average signal gain of each DOX concentration from the data obtained at the three electrodes, and then performed curve fitting for this average signal gain data after plotting versus DOX concentration (Figure S5). We used this curve for the calibration of DOX concentration from the signal gain in all ex vivo and in vivo measurements described throughout the manuscript.

(A)

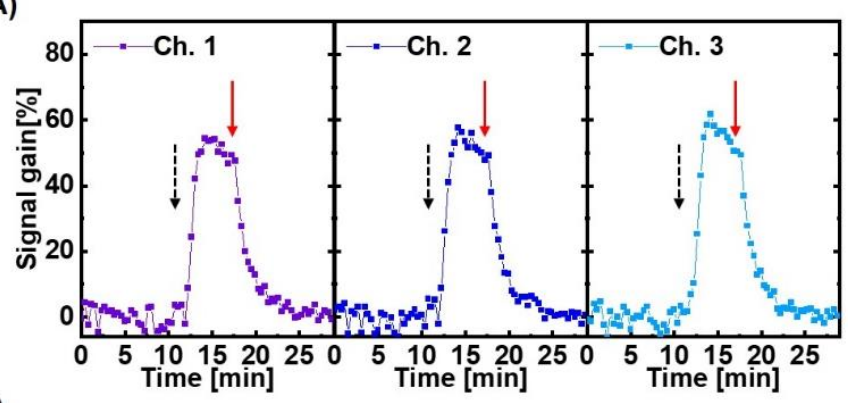

(B)

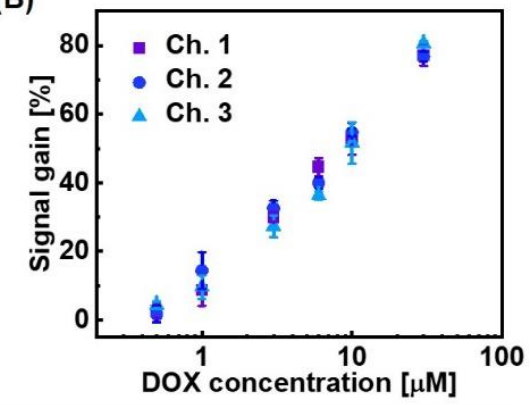

(C)

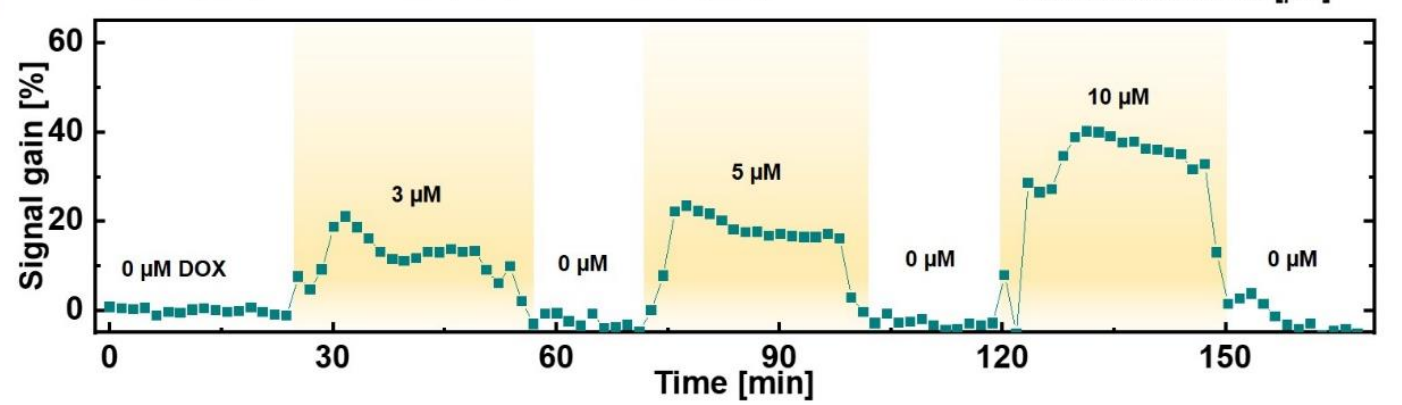

Figure 2. Assessing the consistency and reproducibility of microelectrode array measurements. (A) Continuous measurements of signal gain from each of our three channels at baseline, after adding a $10 \mu \mathrm{M}$ bolus of DOX at $\mathrm{t}=12$ min (black dotted arrow), and after washing in 1x SSC buffer at $\mathrm{t}=17 \mathrm{~min}$ (red solid arrow). (B) Signal gain from all three microelectrode channels at increasing DOX concentrations. Error bars were calculated from five different data points at each concentration. (C) Continuous measurements of signal gain in flowing fetal bovine serum containing different concentrations of DOX. SWV frequency $=200 \mathrm{~Hz}$, amplitude $=50 \mathrm{mV}$. The data represent average signal gain from the continuously-measured signal gain data collected at the three channels.

We next set out to characterize the performance of our sensor in fetal bovine serum. We positioned our microelectrode array sensor vertically within a PDMS chamber, through which we continuously flowed undiluted serum with a peristaltic pump (Figure S6). We increased the DOX concentration in serum to $3 \mu \mathrm{M}, 5 \mu \mathrm{M}$, or $10 \mu \mathrm{M}$ at different time-points, and maintained each condition for $30 \mathrm{~min}$. The aptamer-functionalized microelectrodes clearly responded to each DOX concentration, producing signal gains ranging from $13.2 \%$ at $3 \mu \mathrm{M}$ DOX to $38.8 \%$ 
at $10 \mu \mathrm{M}$ DOX (Figure 2C). Importantly, the sensor consistently returned to baseline when DOX was no longer present in serum, even after nearly three hours of continuous data collection - a time-scale that is standard for clinical DOX administration.

(A)

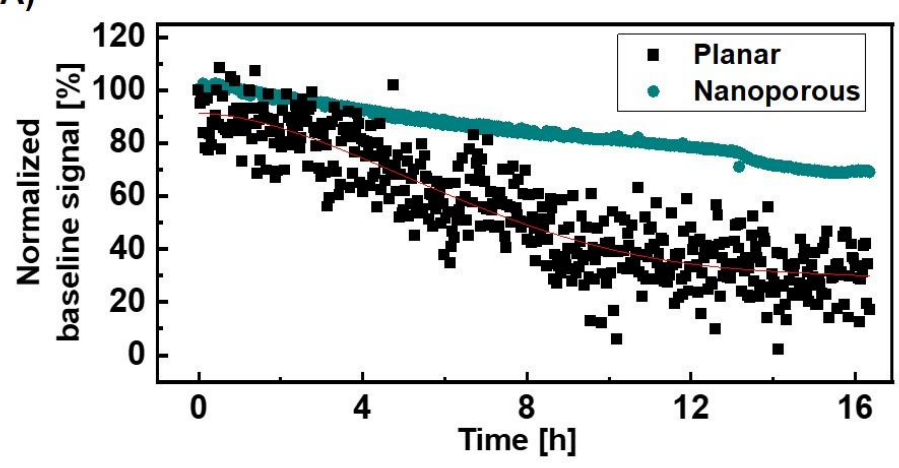

(C)

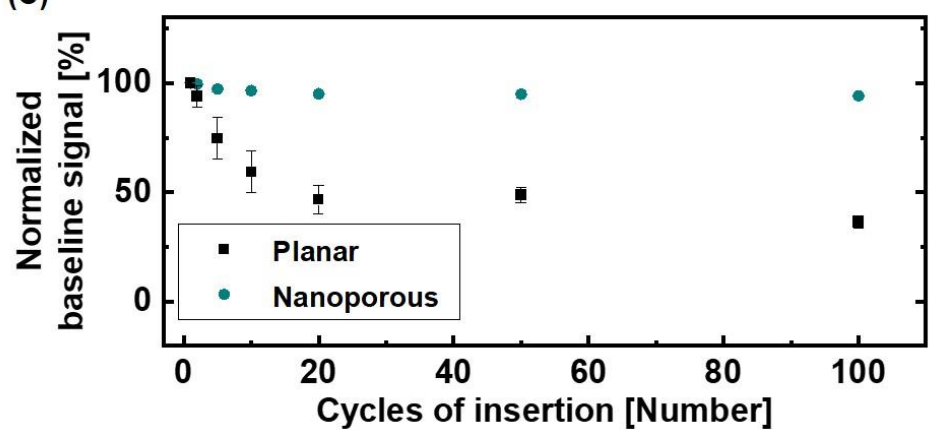

(B)

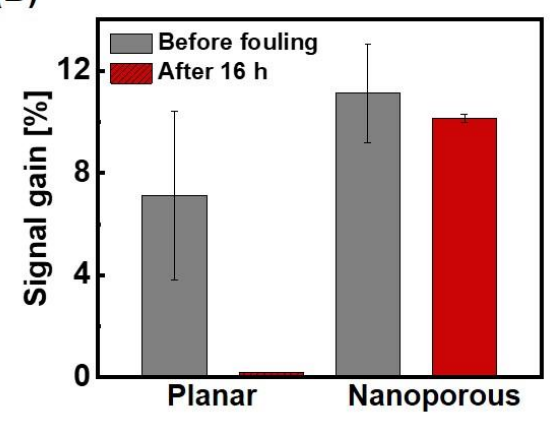

(D)

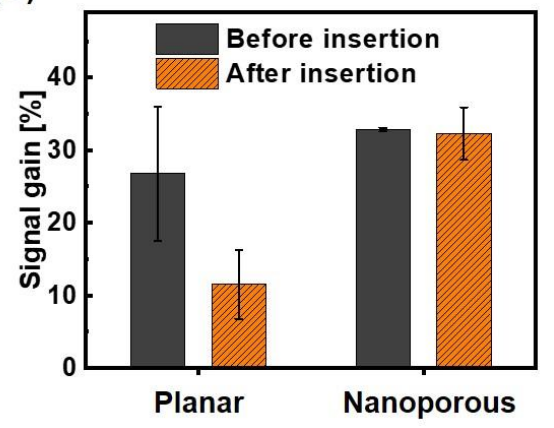

Figure 3. Stability of sensor performance in serum and tissue. (A) Representative data from a single channel showing baseline signal on gold nanoporous and planar microelectrodes over the course of $16 \mathrm{~h}$ in undiluted fetal bovine serum. (B) Average signal gain ( $\mathrm{n}=2$ channels) produced by gold nanoporous and planar microelectrodes in the presence of $3 \mu \mathrm{M}$ DOX in serum upon initial exposure (gray) and after $16 \mathrm{~h}$ in serum (red). (C) Averaged baseline signal change ( $\mathrm{n}=3$ channels) for gold nanoporous and planar microelectrodes after multiple cycles of insertion into murine melanoma tumor tissue. (D) Averaged signal gain ( $n=3$ channels) from $6 \mu \mathrm{M}$ DOX in 1x SSC on planar and nanoporous microelectrodes before initial insertion into tumor tissue and after 100 insertion cycles. SWV frequency $=200 \mathrm{~Hz}$, Amplitude $=50 \mathrm{mV}$.

Biofouling of electrode surfaces poses a major problem for in vivo detection, and the adsorption of proteins present in the blood around and inside tumor tissue can render sensors unusable after a short period. Previous aptamer-based sensors have used a passivation layer on the electrode surface to mitigate this problem, but even with such measures, most aptamerbased sensors to date have reported a functional lifetime of no more than 12 hours in flowing blood. ${ }^{40-42}$ Based on prior work with nanostructured gold microelectrodes ${ }^{34,35}$, we anticipated that our sensor would enable stable, long-term monitoring due to sequestration of the aptamers within nanopores, minimizing the effects of biofouling. To verify this, we measured the baseline signal of both planar and nanoporous microelectrodes in flowing serum for $16 \mathrm{~h}$. As expected, 
the signal from planar microelectrodes worsened over time, and decreased to $10 \%$ of the baseline signal by $16 \mathrm{~h}$, indicating severe biofouling and/or degradation of the aptamer (Figure 3A). In contrast, the nanoporous microelectrodes maintained $70 \%$ of the baseline signal after $16 \mathrm{~h}$, indicating much more stable sensor performance in conditions that are highly conducive to biofouling. We next assessed the signal gain produced in response to a $3 \mu \mathrm{M}$ DOX spike in serum - a standard clinical dose - at time zero versus after $16 \mathrm{~h}$ exposure to serum. At initial exposure, the nanoporous microelectrode produced an average signal gain of $11.1 \%$ versus 7.1\% for the planar microelectrodes (Figure 3B and S7). After $16 \mathrm{~h}$, the signal gain from the nanoporous electrodes decreased only slightly to $10.1 \%$ at $3 \mu \mathrm{M}$ DOX, whereas the planar electrode sensor no longer produced a measurable signal gain. These results strongly suggest that the nanoporous gold microelectrodes are far less susceptible to biofouling and/or degradation of the DOX aptamer, and therefore better suited for long-term in vivo measurements.

\section{Measurements from gold nanoporous microelectrodes inserted into tissue}

We also anticipated that our nanoporous structured electrodes would offer protection against mechanical damage to the sensor surface, which could otherwise result in unstable and reduced signal after insertion. For comparative purposes, we fabricated three-channel planar microelectrode array sensors that were otherwise identical to our nanoporous microelectrode array sensors. We inserted planar and nanoporous gold microelectrode array sensors into tumor tissue and analyzed changes in the baseline signal over the course of multiple cycles of insertion. For these experiments, we used tumor tissue derived from the murine B16-F10 melanoma model, which was grown subcutaneously in the hind flank of a C57BL/6 mouse. ${ }^{43}$ In each insertion cycle, the sensor was inserted into tumor tissue and withdrawn immediately. The signal was then measured in 1x SSC buffer after each cycle of insertion. After 10 cycles, the average signal from planar microelectrodes decreased to $59.4 \%$ of the original baseline signal, and after 100 cycles, this signal was reduced to $36.4 \%$ of baseline (Figure 3C). In contrast, our nanoporous microelectrodes retained $94.1 \%$ of their baseline signal even after 100 cycles of insertion. These results clearly demonstrate that the gold nanoporous microelectrodes are very well suited for electrochemical detection in the context of solid tissue, with minimal mechanical damage after insertion to either the substrate or the functionalized aptamers.

We further confirmed this result by comparing the signal gain in response to $6 \mu \mathrm{M} \mathrm{DOX}$ in 1x SSC for these various microelectrodes before insertion into tumor tissue and after 100 cycles of insertion (Figure 3D). The planar and nanoporous microelectrodes respectively 
showed comparable average signal gain of $26.8 \%$ and $32.8 \%$ before insertion. But after 100 cycles, the signal gain from the planar microelectrodes sharply decreased to $11.5 \%$, whereas the nanoporous electrode still maintained a 32.3\% signal gain. We also assessed how well our probe performed in the context of tissues with different mechanical properties, and found that our nanoporous microelectrode sensor retains its baseline signal independent of the elastic modulus of the tissue environment (Figure S8), confirming the broad mechanical compatibility of our sensor design.

\section{Ex vivo real-time monitoring of DOX}

We next assessed the real-time, multi-channel DOX detection capabilities of our sensor in surgically-removed tumor tissue (Figure 4A). We extracted $\sim 100 \mathrm{~mm}^{2}$ of B16-F10 tumor tissue grown in a C57BL/6 mouse. After transferring the tumor tissue to a PDMS chamber within a small volume of buffer, we implanted our sensor into the middle of the tumor tissue such that all three channels of the array were embedded, with channel 1 closest to the center of the tissue. We then injected a $5 \mu \mathrm{g} / \mathrm{g}$ bolus of DOX adjacent to channel 3 and measured the DOX concentration at the three microelectrode channels (Figure 4B). All three clearly responded to this DOX spike in real-time, but each channel detected a different concentration. Channel 3, which was closest to the injection site, detected an average $\sim 2.4 \mu \mathrm{M}$ DOX, whereas the more distal channels 1 and 2 detected much lower average concentrations of $\sim 0.8$ and 0.7 $\mu \mathrm{M}$, respectively. We next injected $500 \mu 1 \mathrm{x}$ SSC buffer three times into the tumor to wash the drug out completely, and this treatment returned the measured concentration to zero. These results demonstrate that our system can discriminate spatial differences in the drug concentration profile at different positions within the tumor tissue. 
(A)

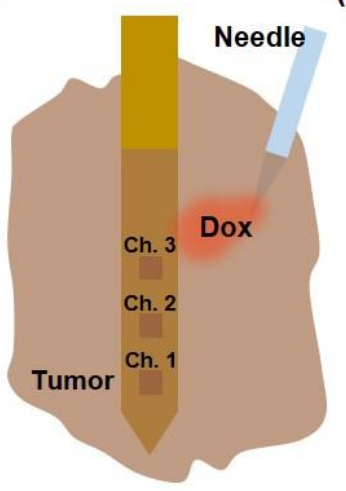

(B)

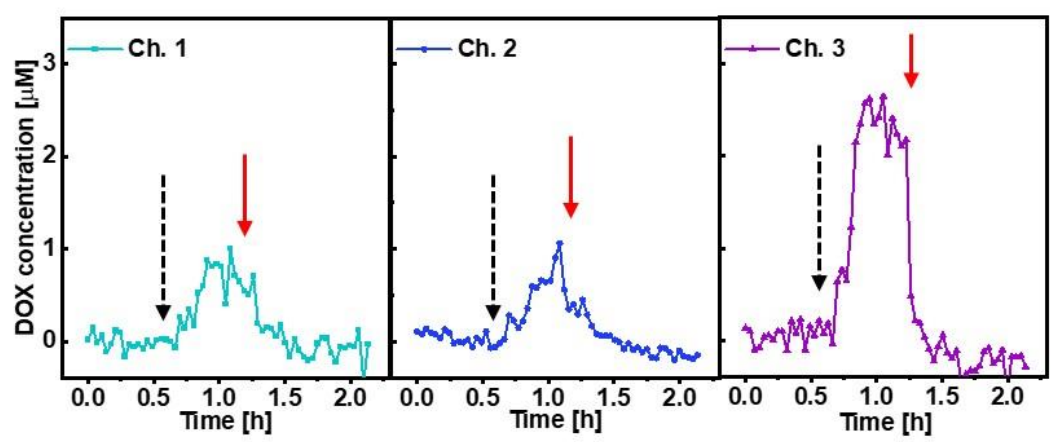

(C)

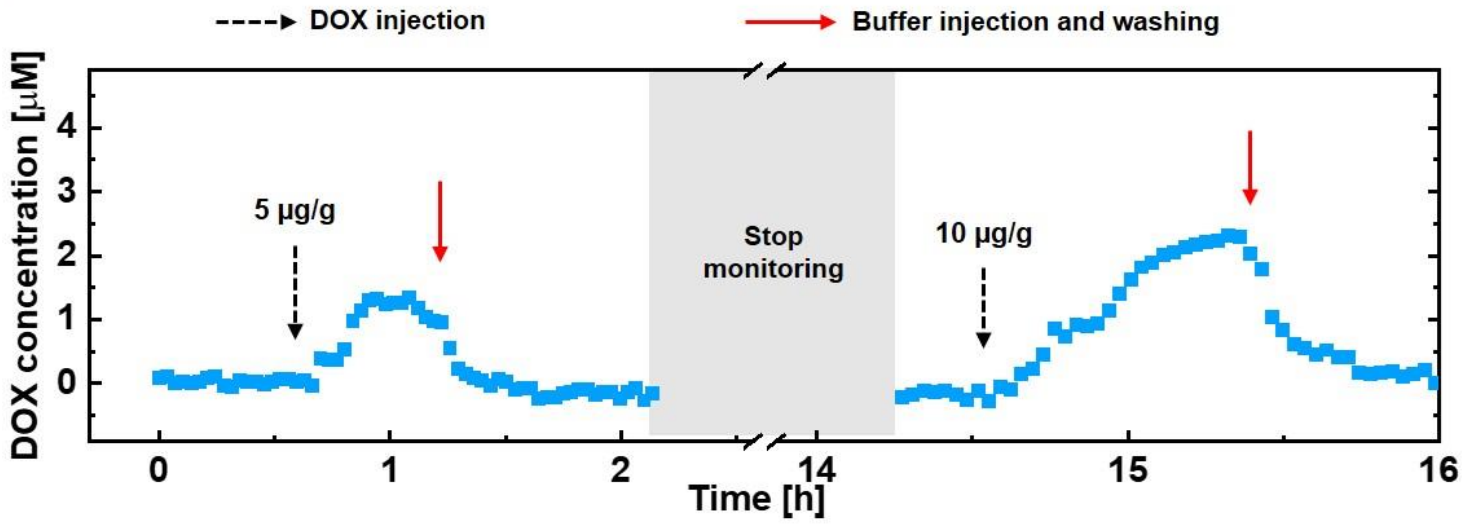

Figure 4. Ex vivo monitoring of DOX in tumor tissue. (A) The sensor was implanted into extracted tumor tissue, after which a bolus of DOX was injected. (B) Real-time DOX concentration at each channel after injecting a $5 \mu \mathrm{g} / \mathrm{g}$ bolus of DOX (black dotted arrow) at a tumor site near channel 3, followed by washing with 1x SSC buffer (red solid arrow). (C) Averaged real-time DOX concentrations from the three sensor channels in tumor tissue over the course of $16 \mathrm{~h}$ after spiking in boluses of $5 \mu \mathrm{g} / \mathrm{g}$ or $10 \mu \mathrm{g} / \mathrm{g}$ DOX at different time-points. $\mathrm{SWV}$ frequency $=200 \mathrm{~Hz}$, amplitude $=50 \mathrm{mV}, \mathrm{n}=3$ channels.

We were also able to achieve continuous detection for extended periods of time within tumor tissue. We injected boluses of DOX at a tumor site adjacent to the sensor at different time-points and monitored the DOX concentration over the course of $16 \mathrm{~h}$ (Figure 4C). After applying the first $5 \mu \mathrm{g} / \mathrm{g}$ bolus, we recorded a peak DOX concentration of $1.3 \mu \mathrm{M}$, which returned to zero after washing the tumor tissue with SSC. After signal stabilization, we stopped monitoring for $12 \mathrm{~h}$ but left the device implanted; when we restarted monitoring, the signal remained at baseline. We subsequently observed a peak DOX concentration of $2.3 \mu \mathrm{M}$ after injecting a second bolus of $10 \mu \mathrm{g} / \mathrm{g}$ DOX at the same site in the tumor. These results demonstrate that our sensor system can achieve robust and stable drug detection even after extended implantation. 


\section{In vivo real-time monitoring of $\mathrm{DOX}$ within tumor tissue}

Finally, we demonstrated the capability to continuously measure drug PK within tumor tissue in a live mouse. We anesthetized a C57BL/6 mouse, which had a 12-mm-diameter B16F10 tumor, and implanted our sensor such that all three channels were within the tumor tissue, with channel 3 closest to the surface and channel 1 deepest within the tumor tissue (Figure S9). After measuring baseline signal within the tumor tissue, we injected a $10 \mu \mathrm{g} / \mathrm{g}$ bolus of DOX adjacent to channel 1 and observed the response at all three channels to assess its in vivo realtime recording capabilities (Figure S10). Channel 1 and 2 responded quickly to this DOX injection, while channel 3 did not respond as it was too far from the injection site for measurable quantities of the drug to diffuse.

We next anesthetized a second C57BL/6 mouse, which also had a 12-mm-diameter B16F10 tumor, and implanted another sensor in the same manner described above. We collected blood from the tail vein using a heparinized capillary tube and inserted an additional third sensor inside the tube. After measuring the baseline signal within the tumor tissue and circulation, we intravenously (i.v.) injected $10 \mu \mathrm{g} / \mathrm{g}$ DOX through the tail vein. We observed different DOX concentration profiles from each of the three channels in the intratumoral probe (Figure 5A), indicating that the drug PK exhibits considerable site-dependent variability due to factors including irregular microvasculature density and interstitial fluid pressure across the whole of the tumor tissue. ${ }^{15,44-46}$ In parallel, we collected blood samples from the tail vein at 0, 5, 30, 60, and 120 min time-points after the i.v. injection to measure circulatory PK. We derived the PK parameters by fitting to a two-compartment model, which describes the changes in drug concentrations in the central (i.e. systemic circulation) and peripheral (i.e. tumor tissue) compartments (Table 1). ${ }^{25,47,48}$ From the perspective of the circulation, this model describes the rate of transport of the drug into the tumor ( $\left.\mathrm{K}_{\mathrm{d}_{-} \text {blood }}\right)$ as well as the subsequent rate of drug elimination $\left(\mathrm{K}_{\mathrm{el}}\right)$ from the body. For the tumor tissue, we derived the PK parameters by fitting to an one-compartment model, which describes the rate of entry of the drug into a particular region of the tumor from the blood $\left(\mathrm{K}_{\mathrm{a}_{-}}\right.$tumor $)$as well as the rate of drug elimination $\left(\mathrm{K}_{\mathrm{el}}\right)$ from the tumor.

Channel 1 was nearest the center of the tumor, where the high interstitial fluid pressure due to the dense microvasculature results in a lower rate of drug penetration relative to the surface of tumor tissue. ${ }^{49,50}$ Accordingly, we began to observe DOX signal in channel $130 \mathrm{~min}$ after i.v. injection, followed by a relatively slow increase in drug concentration $\left(\mathrm{K}_{\mathrm{a}_{-} \text {tumor }}=0.05\right.$ $\left.\min ^{-1}\right)$. We observed a much earlier response in channels 2 and 3-18 min and 15 min after i.v. injection, respectively — and much higher $\mathrm{K}_{\mathrm{a}_{-} \text {tumor }}\left(0.077 \mathrm{~min}^{-1}\right.$ and $0.095 \mathrm{~min}^{-1}$, respectively). 
(A)

(C)

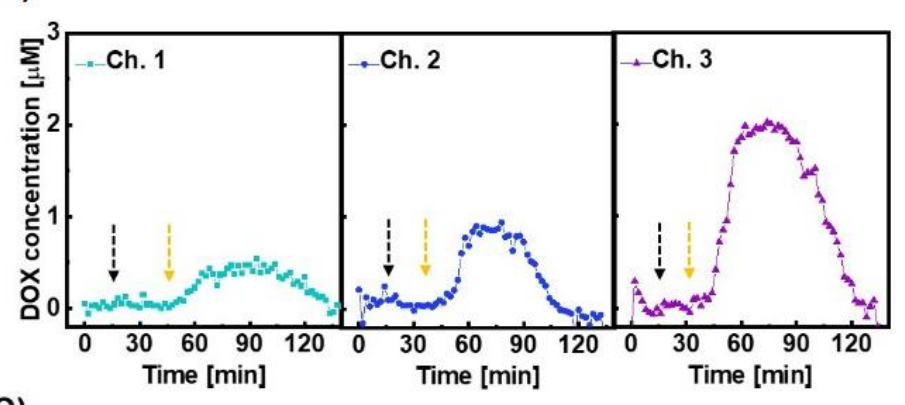

(B)

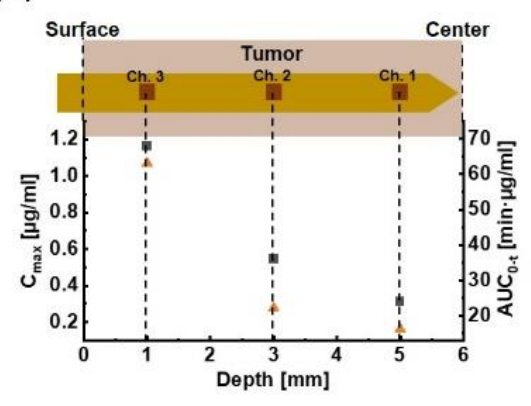

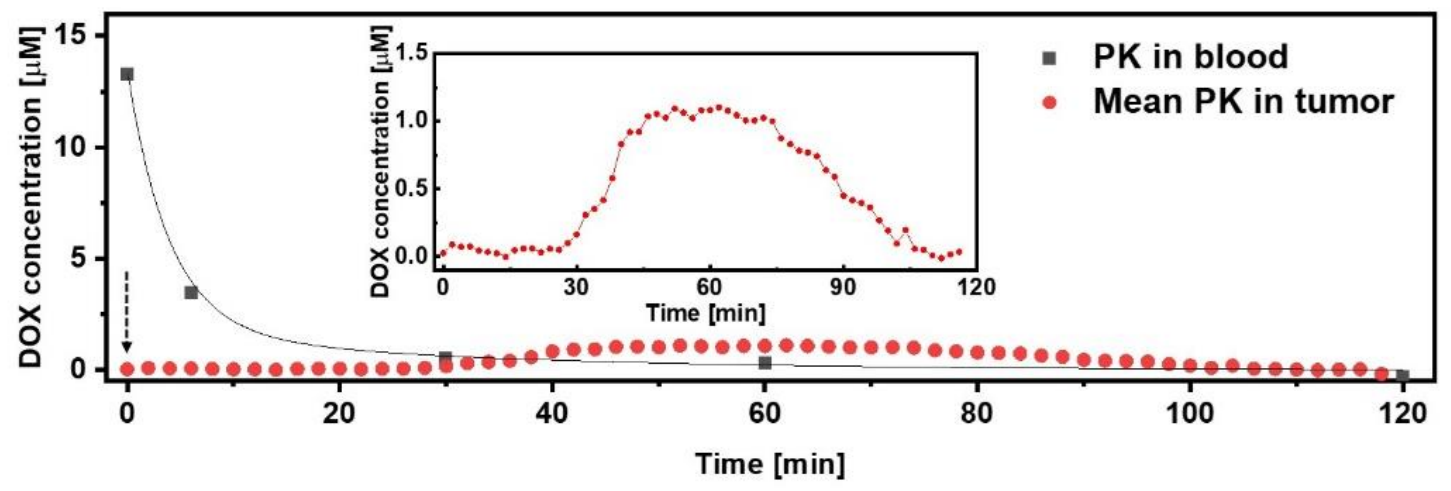

Figure 5. In vivo real-time monitoring of DOX PK in tumor tissue. (A) Real-time DOX concentration at each sensor channel after intravenous (i.v.) injection of $10 \mu \mathrm{g} / \mathrm{g}$ DOX at $\mathrm{t}=15$ min (black dotted arrow). Yellow dotted arrows represent the time-points that the response is observed. (B) Maximum drug concentration $\left(\mathrm{C}_{\max }\right)$ (black square) and area under the curve $\left(\mathrm{AUC}_{0-\mathrm{t}}\right)$ (orange triangle) as a function of depth within the tumor. (C) Averaged real-time DOX concentrations from the three sensor channels versus DOX concentrations obtained from blood after injecting $10 \mu \mathrm{g} / \mathrm{g}$ DOX at $\mathrm{t}=0 \mathrm{~min}$ (black dotted arrow). Line represents curve fitting to a two-compartment pharmacokinetic model. Inset is magnified averaged real-time intratumoral DOX concentration data. For (A) and (C), SWV frequency $=200 \mathrm{~Hz}$, amplitude $=50 \mathrm{mV}$.

We observed a clear gradient of decreasing drug exposure as we looked deeper into the tumor (Figure 5B). For example, the maximum drug concentration $\left(\mathrm{C}_{\max }\right)$ for channel $1(0.312$ $\mu \mathrm{g} / \mathrm{ml}$ ), which is located at a $5-\mathrm{mm}$ depth within the tumor, was four times lower than for channel $3(1.163 \mu \mathrm{g} / \mathrm{ml})$, which is positioned at a 1-mm depth. Similarly, the area under the curve $\left(\mathrm{AUC}_{0-\mathrm{t}}\right)$, which reflects the total drug exposure over time, gradually decreased from $63.057 \mathrm{~min} \cdot \mu \mathrm{g} / \mathrm{ml}$ to $16.159 \mathrm{~min} \cdot \mu \mathrm{g} / \mathrm{ml}$ as we sampled deeper within the tumor, which would presumably lead to the differential therapeutic efficacy of chemotherapy across the tumor. In parallel, each channel reached $\mathrm{C}_{\max }$ at an increasingly later time-point post-administration $\left(\mathrm{T}_{\max }\right)$ as we looked deeper and deeper into the tumor (Figure S11). Interestingly, we noted that the PK parameters did not always fall along a clear continuum from channel 1 to 2 to 3 . For example, we measured a lower elimination half-life $\left(\mathrm{t}_{1 / 2}\right)$ at channel 2 (82 $\left.\mathrm{min}\right)$ than channel 3 (90 $\left.\mathrm{min}\right)$, even though the drug concentration at channel 2 reached $C_{\max }$ later than channel $3\left(\mathrm{~T}_{\max }=63\right.$ min versus $59 \mathrm{~min})$. We posit that this is due to the fact that the DOX elimination rate $\left(\mathrm{K}_{\mathrm{el}}\right)$ was 
higher in the tissue surrounding channel $2\left(0.067 \mathrm{~min}^{-1}\right)$ compared to at channel $3\left(0.04 \mathrm{~min}^{-1}\right)$. These results reveal how the PK can vary across the tumor tissue in a manner that would be difficult or impossible to measure accurately based on conventional biopsy methods.

Table 1. Pharmacokinetic parameters in tumor and blood.

\begin{tabular}{c|c|cccc}
\hline PK parameter** & In blood & Ch. 1 & Ch. 2 & Ch. 3 & Mean PK \\
\hline AUC $_{0-\mathrm{t}}[\mathrm{min} \cdot \mu \mathrm{g} / \mathrm{ml}]$ & 80.915 & 16.159 & 22.147 & 63.057 & 33.376 \\
$\mathrm{C}_{\max }[\mu \mathrm{gg} / \mathrm{ml}]$ & 7.702 & 0.312 & 0.546 & 1.163 & 0.625 \\
$\mathrm{~T}_{\max }[\mathrm{min}]$ & 0 & 79 & 63 & 59 & 64 \\
$\mathrm{t}_{1 / 2}[\mathrm{~min}]$ & 3.4 & 101 & 82 & 90 & 89 \\
$\mathrm{~K}_{\text {el }}[1 / \mathrm{min}]$ & $0.138 \pm 0.08$ & $0.041 \pm 0.005$ & $0.067 \pm 0.007$ & $0.04 \pm 0.004$ & $0.039 \pm 0.004$ \\
$\mathrm{~K}_{\text {a_tumor }}[1 / \mathrm{min}]$ & $\mathrm{N} / \mathrm{A}$ & $0.05 \pm 0.007$ & $0.077 \pm 0.009$ & $0.095 \pm 0.009$ & $0.076 \pm 0.009$ \\
$\mathrm{~K}_{\text {d_blood }}[1 / \mathrm{min}]$ & $0.064 \pm 0.094$ & $\mathrm{~N} / \mathrm{A}$ & $\mathrm{N} / \mathrm{A}$ & $\mathrm{N} / \mathrm{A}$ & $\mathrm{N} / \mathrm{A}$ \\
\hline
\end{tabular}

** $\mathrm{AUC}_{0-\mathrm{t}}$ is the area under the curve until the drug concentration reaches zero; $\mathrm{C}_{\max }$ is the maximum drug concentration; $\mathrm{T}_{\max }$ is the time at $\mathrm{C}_{\max } ; \mathrm{t}_{1 / 2}$ is the elimination half-life; $\mathrm{K}_{\mathrm{el}}$ is the elimination rate of drug; $\mathrm{K}_{\mathrm{a} \_ \text {tumor }}$ is the absorption rate of the drug within the tumor; and $\mathrm{K}_{\mathrm{d} \_ \text {blood }}$ is the distribution rate of the drug from the bloodstream to the tissue.

Finally, we showed that the PK measurements obtained from the tumor tissue differ markedly from the systemic PK (Figure 5C and Table 1). We made this comparison by determining the mean values for the various PK parameters within the tumor tissue by fitting the average real-time DOX concentrations at all three channels over the course of the experiment. We observed that the total drug exposure $\left(\mathrm{AUC}_{0-\mathrm{t}}\right)$ in blood $(80.915 \mathrm{~min} \cdot \mu \mathrm{g} / \mathrm{ml})$ was $\sim 2.5$-fold greater than in the tumor $(33.376 \mathrm{~min} \cdot \mu \mathrm{g} / \mathrm{ml})$, indicating that the tumor received only a fraction of the drug circulating in the blood. Likewise, $C_{\max }$ in blood $(7.702 \mu \mathrm{g} / \mathrm{ml})$ was over ten-fold greater than in tumor tissue $(0.625 \mu \mathrm{g} / \mathrm{ml})$, which raises the possibility of suboptimal drug dosing if one was to rely entirely on PK data from the bloodstream. The $\mathrm{t}_{1 / 2}$ and $\mathrm{K}_{\mathrm{el}}$ values were respectively very short $(3.4 \mathrm{~min})$ and fast $\left(0.138 \mathrm{~min}^{-1}\right)$ in the blood, reflecting rapid clearance by renal excretion. ${ }^{51}$ In contrast, elimination was considerably slower within the tumor $\left(\mathrm{t}_{1 / 2}=89 \mathrm{~min}, \mathrm{~K}_{\mathrm{el}}=0.039 \mathrm{~min}^{-1}\right)$. This indicates that the drug remains present and active within this tissue for a longer duration, which could result in an overdose if one were to rely solely on PK measurements from the blood. Conversely, this slower elimination rate could result in a more potent effect even from a seemingly low dose, which is a useful consideration when attempting to identify the appropriate therapeutic window. This striking variability in PK parameters between blood and tumor demonstrates the importance of obtaining local measurements of drug absorption within tumor tissue, and shows how in situ tumor measurements collected with our sensor could facilitate the selection of more effective and 
appropriate drug dosing strategies.

\section{Conclusion}

Drug PK can vary considerably within cancerous tissue relative to the systemic circulation due to the abnormal physiology of the tumor microenvironment. As a consequence, blood-based measurements of drug absorption and elimination are likely to produce misleading or inaccurate PK measurements that impede efforts to identify an optimally safe and effective dose for cancer therapeutics. In this work, we present a microelectrode array-based implantable electrochemical aptamer sensor that overcomes this problem by enabling simultaneous multisite drug concentration monitoring within tumor tissue in real-time. Our sensor incorporates gold nanoporous electrodes that are highly resistant to both damage and biofouling during or after tissue implantation, and is built on a flexible polymer substrate that matches the physical properties of the surrounding tissue and thus minimizes tissue disruption at the site of insertion. Most importantly, each probe contains three distinct microelectrode channels, and we demonstrate the capacity to sensitively discriminate local differences in the intratumoral concentration of DOX at each channel. Our sensor enabled us to collect extensive tumorspecific PK data over the course of multiple hours that reveal striking differences in the profile of DOX absorption and elimination relative to PK measurements based on systemic circulation from the same animal.

Based on these findings, we believe that our sensor platform could offer a highly effective tool for preclinical analysis of the PK characteristics of experimental drugs, thereby guiding dose selection for first-in-human studies that maximize likelihood of efficacy while minimizing dose-related toxicity. The foundational sensor design demonstrated here should be readily extensible to include larger numbers of channels that can produce measurements with even greater spatial resolution. In principle, different microelectrode channels could also be functionalized with different aptamers, enabling the real-time monitoring of multiple drug agents in the context of a combination therapy, or simultaneous measurement of a therapeutic agent and a biomarker related to drug response. With further refinement and demonstration of the long term stability, safety, and biocompatibility of this probe design, we could envision future adaptations of this platform for potential use in clinical drug and biomarker studies. 


\section{METHODS}

\section{Reagents and materials}

The DOX aptamer was obtained from Biosearch Technologies: 5'-HS-C6ACCATCTGTGTAAGGGGTAAGGGGTGGT-MB-3', where MB indicates the methylene blue redox reporter. Tris(2-carboxyethyl)phosphine (TCEP), doxorubicin (DOX), and 6-mercapto-1-hexanol (6$\mathrm{MCH}$ ) were purchased from Sigma-Aldrich. A diluted 1X saline-sodium citrate (SSC) buffer was prepared by diluting 20X SSC buffer (Thermo Fisher) with nuclease-free water. Fetal bovine serum (FBS) was obtained from Thermo Fisher. Solutions of various DOX concentrations were prepared by dissolving DOX in either 1X SSC buffer or undiluted FBS. The conventional $\mathrm{Ag} / \mathrm{AgCl}$ reference electrode was prepared from 500- $\mu$ m-diameter Ag wire (41390 Silver wire, Alfa Aesar) treated with 1 M iron(III) chloride (Sigma-Aldrich) solution for $1 \mathrm{~min}$.

\section{Fabrication of microelectrode array sensor}

A schematic of the device fabrication process is shown in Figure S2. For the planar gold microelectrodes, a 300-nm-thick aluminum ( $\mathrm{Al}$ ) sacrificial layer was deposited on a $\mathrm{Si}$ wafer by electron-beam evaporation (ATC-E, AJA International Inc.). A 15- $\mu$ m-thick polyimide (PI) layer (PI2574, HD MicroSystems) was spin-coated onto the Al layer by manual resist spinner (Headway Research) and thermally imidizated by baking in a $\mathrm{N}_{2}$-purged oven at $250{ }^{\circ} \mathrm{C}$ for $2 \mathrm{~h}$ and subsequently cooling down to room temperature RT for $4 \mathrm{~h}$. Then, a 2- $\mu$ m-thick negative photoresist (PR) layer (NR9-3000PY, Futurrex) was spin-coated onto the PI layer, and then patterned by photolithography with a contact mask aligner (Karl Suss MA-6, SUSS MicroTec) to make the pattern of a $100 \times 100 \mu \mathrm{m}^{2}$ three-channel array. Subsequently, a Ti/Au $(5 \mathrm{~nm} / 70 \mathrm{~nm})$ layer was deposited onto the PR layer using electron-beam evaporation, after which the gold planar microelectrode array was formed through a lift-off process in which the PR layer was dissolved in acetone.

For the nanoporous microelectrode arrays, a 4.5 - $\mu \mathrm{m}$-thick positive PR layer (MEGAPOSIT SPR220-3, Kayaku Advanced Materials) was subsequently spin-coated onto the sample. This was patterned to make the pattern of a $100 \times 100 \mu \mathrm{m}^{2}$ three-channel array as described above. A Ti/Au (10 $\mathrm{nm} / 50 \mathrm{~nm}$ ) bottom-protective layer (BPL) was then deposited onto the PR via sputter deposition (LAB Line SPUTTER, Kurt J. Lesker Co.). A 300-nm-thick Au:Ag alloy layer was then deposited by cosputtering $\mathrm{Au}$ and $\mathrm{Ag}$. The alloy was composed of $66.7 \% \mathrm{Ag}$ and $33.3 \% \mathrm{Au}$. After deposition, the sample was immersed in $69 \% \mathrm{v} / \mathrm{v}$ nitric acid for $7 \mathrm{~min}$ at $\mathrm{RT}$ to dissolve the $\mathrm{Ag}$, forming a gold nanoporous layer. A lift-off process was then used to form the final gold nanoporous microelectrode array.

For both sensor designs, a 2- $\mu$ m-thick SU8 encapsulation layer (SU8-2002, Microchem) was spin-coated onto the sample, and then patterned by photolithography with the contact mask aligner to encapsulate the entire sensor with the exception of the microelectrode array. This assembly was then dry-etched with an ICP-RIE etcher (Versaline LL ICP, Plasma-Therm) and Al etching mask to define the shape of the sensor. After removal of the $\mathrm{Al}$ etching mask, the sensor was released from the wafer 
through anodic dissolution of the Al sacrificial layer. For this step, the Si wafer was connected to a DC power supply (1666, B\&K Precision), immersed in $2 \mathrm{M} \mathrm{NaCl}$, and $15 \mathrm{~V} \mathrm{DC}$ voltage was applied to the Al layer.

\section{Functionalization of aptamer onto the electrode}

The DOX aptamer was dissolved in nuclease-free water with a concentration of $100 \mu \mathrm{M}$. This solution was reacted with a 1,000-fold molar excess of TCEP solution with a 1:1 volume ratio for $1 \mathrm{~h}$, leading to reduction of the MB moiety and thiol-end group on the aptamer. Afterwards, the freshly-prepared sensor was rinsed with DI water, and then functionalized with $1 \mu \mathrm{M}$ of the TCEP-treated DOX aptamer in $1 \mathrm{x}$ SSC buffer for $2 \mathrm{~h}$ at RT. The sensor was then washed with excess buffer and incubated with $7 \mathrm{mM} 6-$ $\mathrm{MCH}$ solution for $24 \mathrm{~h}$ at RT to passivate the remaining electrode surface. The sensor was stored in $1 \mathrm{x}$ SSC at $4{ }^{\circ} \mathrm{C}$ until it was used for electrochemical measurement.

\section{Electrochemical signal measurement}

All in vitro measurements were performed in a PDMS chamber with the probe connected to a potentiostat (PalmSens4, PalmSens) and multiplexer (MUX8-R2, PalmSens). The PDMS chamber was made by punching a 6-mm-diameter hole on the 5-mm-thick PDMS film and subsequently putting the PDMS film on a glass slide. We used two types of PCB, one small and one large. Each was soldered with an 8-pin FPC connector (FH19C-8S-0.5SH, Hirose Electric Co.). The other side of the large PCB was soldered to another connector (NPD-FF, Omnetics Connector Corporation). The sensor was connected to the small PCB, which was in turn connected to the large PCB. A wire connector (NSDWD, Omnetics Connector Corporation) connected to the large PCB linked the three channels on the sensor to the commercial potentiostat. We placed the sensor and $\mathrm{Ag} / \mathrm{AgCl}$ reference electrode into the PDMS chamber and adjusted the height to completely submerges all three channels while avoiding direct contact between the connector and the buffer solution. The PDMS chamber was filled with $1 \mathrm{x}$ SSC buffer. Square wave voltammetry (SWV) measurement was carried out over the potential range of $-0.55 \mathrm{~V}$ to $-0.1 \mathrm{~V}$ with an amplitude of $50 \mathrm{mV}$, step size of $1 \mathrm{mV}$, and pulse frequencies of $200 \mathrm{~Hz}$. Data processing and visualization were performed with custom MATLAB code. All modules were connected as shown in Figure $\mathbf{S 4}$.

Continuous in vitro drug monitoring experiments and biofouling tests in undiluted FBS were conducted in a flowing system that switched between FBS only and FBS plus DOX. Flow was achieved by connecting the PDMS chamber with a peristaltic pump, as shown in Figure S6. The inlet and outlet were connected through fluorinated ethylene propylene tubes (Tygon tubing, $1.58 \mathrm{~mm}$ inside diameter), which were then mounted onto the peristaltic pump, with a flow-rate of $100 \mu 1 / \mathrm{min}$.

\section{Melanoma model}


All animal studies were performing in accordance with Stanford's IACUC guidelines and protocols (APLAC protocol \# 32947). Murine B16F10 melanoma cells were purchased from ATCC, tested for mycoplasma contamination using the MycoAlert Microplasma Kit (Lonza), and cultured with 0.2micron filtered DMEM media (Thermo Fisher) supplemented with 10\% FBS (Novus Biologicals) and $1 \%$ penicillin-streptomycin (Thermo Fisher). C57BL/6 mice (7-8 weeks old; Charles River Laboratories) were subsequently subcutaneously injected with $100 \mu \mathrm{L}$ of $3 \times 10^{6}$ B16F10 cells $/ \mathrm{mL}$ in PBS above the right hind leg. Following tumor inoculation, mice were monitored for the formation of palpable tumors, which occurred within 7-10 days. Tumors were regularly monitored via caliper (Mitotoyu) measurements until they reached the appropriate size for either ex vivo or in vivo studies. Mice were euthanized $\left(\mathrm{CO}_{2}\right.$ asphyxiation followed by cervical dislocation) when overall tumor area ( $\mathrm{L}$ $\mathrm{x} \mathrm{W}$ ) exceeded $150 \mathrm{~mm}^{2}$, or if mice displayed signs of morbidity (e.g., pain, hunching, ulceration, wasting).

\section{Ex vivo tumor experiments}

Tumors were surgically removed once they grew to $\sim 100 \mathrm{~mm}^{2}$, after euthanasia of the mice. Sensor insertion was tested by inserting the sensor into random positions in the tumor, after which the sensor was immediately withdrawn - this constituted one cycle of insertion. We conducted multiple insertion cycles, and SWV measurements were carried out in 1x SCC buffer after each cycle to monitor sensor function.

DOX detection in ex vivo tissues was assessed by placing excised tumors into the PDMS chamber in a small volume of 1x SSC buffer. The sensor was gently implanted into the middle of the tumor tissue to make sure all three channels were inside the tissue. SWV measurement was carried out over the potential range of $-0.55 \mathrm{~V}$ to $-0.1 \mathrm{~V}$ with an amplitude of $50 \mathrm{mV}$, step size of $1 \mathrm{mV}$, and pulse frequencies of $200 \mathrm{~Hz}$ as we injected boluses of DOX into the tumor. Once the measurement was complete, we left the probe in place but washed out the drug by injecting $500 \mu 1$ of 1x SSC buffer three times into the tumor and collected the waste solution from the PDMS chamber. We then added fresh $1 \mathrm{x}$ SSC buffer to the chamber. After signal stabilization, we stopped SWV measurement but left the experimental set-up in place for $12 \mathrm{~h}$. SWV measurement was then carried out again with a second bolus of DOX and an additional washing process.

\section{In vivo experiments}

Tumors were allowed to grow to a sufficient size to accommodate the sensor $\left(\sim 140 \mathrm{~mm}^{2}\right)$. Mice were then anesthetized using isoflurane (3\% induction, $2 \%$ maintenance) and maintained on a heating pad at $35{ }^{\circ} \mathrm{C}$ in a Faraday Cage (VistaShield, Gamry Instruments). To prevent uneccesary pain or discomfort, mice were subcutaneously injected with buprenorphine-SR $(0.5 \mathrm{mg} / \mathrm{kg})$ and Puralube ophthalmic ointment was applied to the eyes. Analgesics were given $15 \mathrm{~min}$ to take effect prior to beginning the procedure. Our sensor and $\mathrm{Ag} / \mathrm{AgCl}$ reference electrode were vertically implanted into the middle of the 
tumor tissue, and SWV measurement was performed over the potential range of $-0.55 \mathrm{~V}$ to $-0.1 \mathrm{~V}$ with an amplitude of $50 \mathrm{mV}$, step size of $1 \mathrm{mV}$, and pulse frequencies of $200 \mathrm{~Hz}$.. After stabilization of the baseline signal of the sensor, DOX $(10 \mu \mathrm{g} / \mathrm{g})$ was injected through the tail vein manually using a $28 \mathrm{G}$ syringe needle. At the end of the experiments, mice were euthanized.

\section{PK parameter analysis}

The DOX concentration-time curve in blood (Fig. 5C) was fitted to a bi-exponential equation indicating the two-compartment model:

$$
\mathrm{C}_{\text {Doxorubicin }}=A e^{-\alpha t}+B e^{-\beta t}
$$

Where $\mathrm{A}$ and $\mathrm{B}$ are maximum plasma concentrations corresponding to the drug distribution phase and drug elimination phase, respectively, and $1 / \alpha$ and $1 / \beta$ are the half-lives for distribution and elimination, respectively. $\mathrm{C}_{\text {Doxorubicin }}$ is DOX concentration as a function of time.

$\mathrm{K}_{\mathrm{d} \_b l o o d}$ was calculated by Equation 2:

$$
\mathrm{K}_{\mathrm{d} \_ \text {blood }}=\left(\left(\mathrm{A}^{*} \beta\right)+\left(\mathrm{B}^{*} \alpha\right)\right) /(\mathrm{A}+\mathrm{B})
$$

Elimination rate $\left(\mathrm{K}_{\mathrm{el}}\right)$ was calculated by Equation 3:

$$
\mathrm{K}_{\mathrm{el}}=\left(\alpha^{*} \beta\right) / \mathrm{K}_{\mathrm{d} \_ \text {blood }}
$$

The DOX concentration-time curve within the tumor (Fig. 5A, C) were fitted to an exponential equation indicating the one-compartment model:

$$
\mathrm{C}_{\text {Doxorubicin }}=B e^{-\beta t}
$$

Where $\beta$ is $K_{\mathrm{el}}$ of drug within tumor.

$\mathrm{K}_{\mathrm{a}_{-} \text {tumor }}$ was derived by fitting the absorption phase of the DOX concentration-time curve to Equation 5:

$$
\mathrm{C}_{\text {Doxorubicin }}=A e^{(\text {Ka_tumor } * t)^{*}}
$$

The absorption phase is the time range from drug injection to when the drug concentration reaches $\mathrm{C}_{\max } . \mathrm{C}_{\max }, \mathrm{T}_{\max }, \mathrm{t}_{1 / 2}$ were directly obtained from the experimental raw data of the DOX concentrationtime curve. The area under the curve (AUC) was calculated using the definite integral of the DOX concentration-time curve with a time range from drug injection to when the drug concentration reached zero. 


\section{ACKNOWLEDGEMENTS}

This work was supported by the Chan-Zuckerberg Biohub, the Biomedical Advanced Research and Development Agency (BARDA, 75A50119C00051) and the National Institutes of Health (NIH, OT2OD025342). Ji Won Seo was supported by Basic Science Research Program through the National Research Foundation of Korea(NRF) funded by the Ministry of Education (2018R1C1B6009140). S.C. is supported by the National Cancer Institute of the National Institutes of Health under Award Number F32CA247352. We thank Celine Liong and Carolyn K. Jons for their thoughtful assistance on the in vivo experiments. We thank Leighton Wan, Ian Thompson, and Alyssa Cartwright for their thoughtful comments and valuable suggestions.

\section{AUTHOR CONTRIBUTION}

J.-W.S., K.F., and H.T.S. initiated the project and designed experiments. J.-W.S designed and fabricated the sensor. J.-W.S. conducted experiments and analyzed the data. J.-W.S. and S.C. performed animal experiments. J.-W.S., K.F., S.C., E.A.A., and H.T.S. discussed the data. J.W.S., K.F., M.E. and H.T.S. wrote the paper. All authors edited the paper.

\section{Conflict of Interest}

The authors declare no conflict of interest. 


\section{References}

1. Vasey, P.A. et al. Phase I Clinical and Pharmacokinetic Study of PK1 [N-(2Hydroxypropyl)methacrylamide Copolymer Doxorubicin]: First Member of a New Class of Chemotherapeutic Agents - Drug-Polymer Conjugates. Clin. Cancer Res. 5, 83-94 (1999).

2. Szakacs, G., Paterson, J.K., Ludwig, J.A., Booth-Genthe, C. \& Gottesman, M.M. Targeting multidrug resistance in cancer. Nat. Rev. Drug Discov. 5, 219-234 (2006).

3. Monsuez, J.-J., Charniot, J.-C., Vignat, N. \& Artigou, J.-Y. Cardiac side-effects of cancer chemotherapy. Int. J. Cardiol. 144, 3-15 (2010).

4. Jain, R.K. Normalization of Tumor Vasculature: An Emerging Concept in Antiangiogenic Therapy. Science 307, 58-62 (2005).

5. Perazella, M.A. \& Moeckel, G.W. Nephrotoxicity From Chemotherapeutic Agents: Clinical Manifestations, Pathobiology, and Prevention/Therapy. Semin. Nephrol. 30, 570-581 (2010).

6. Kerbel, R.S. \& Kamen, B.A. The anti-angiogenic basis of metronomic chemotherapy. Nat. Rev. Cancer 4, 423-436 (2004).

7. Gurney, H. How to calculate the dose of chemotherapy. Brit. J. Cancer 86, 1297-1302 (2002).

8. Arroyo-Curras, N. et al. Real-time measurement of small molecules directly in awake, ambulatory animals. Proc. Natl. Acad. Sci. U.S.A. 114, 645-650 (2017).

9. Arroyo-Curras, N., Scida, K., Ploense, K.L., Kippin, T.E. \& Plaxco, K.W. High Surface Area Electrodes Generated via Electrochemical Roughening Improve the Signaling of Electrochemical Aptamer-Based Biosensors. Anal. Chem. 89, 1218512191 (2017).

10. Arroyo-Curras, N. et al. Subsecond-Resolved Molecular Measurements in the Living Body Using Chronoamperometrically Interrogated Aptamer-Based Sensors. ACS sensors 3, 360-366 (2018).

11. Ogata, G. et al. A microsensing system for the in vivo real-time detection of local drug kinetics. Nat. Biomed. Eng. 1, 654-666 (2017).

12. Muley, H., Fado, R., Rodriguez-Rodriguez, R. \& Casals, N. Drug uptake-based chemoresistance in breast cancer treatment. Biochem. Pharmacol. 177, 113959 (2020).

13. Maeda, H., Wu, J., Sawa, T., Matsumura, Y. \& Hori, K. Tumor vascular permeability and the EPR effect in macromolecular therapeutics: a review. J. Control. Release 65, 271-284 (2000).

14. Matsumura, Y. \& Maeda, H. A New Concept for Macromolecular Therapeutics in Cancer-Chemotherapy - Mechanism of Tumoritropic Accumulation of Proteins and the Antitumor Agent Smancs. Cancer Res. 46, 6387-6392 (1986).

15. Muller, M., Pena, A.D. \& Derendorf, H. Issues in Pharmacokinetics and Pharmacodynamics of Anti-Infective Agents: Distribution in Tissue. Antimicrob. Agents Ch. 48, 1441-1453 (2004).

16. Peng, P.-C., Hong, R.-L., Tsai, T. \& Chen, C.-T. Co-Encapsulation of Chlorin e6 and Chemotherapeutic Drugs in a PEGylated Liposome Enhance the Efficacy of Tumor Treatment: Pharmacokinetics and Therapeutic Efficacy. Pharmaceutics 11, 617 (2019).

17. Goel, S., Wong, A.H.-K. \& Jain, R.K. Vascular Normalization as a Therapeutic Strategy for Malignant and Nonmalignant Disease. Cold Spring Harb. Perspect. Med. 2, a006486 (2012).

18. Goel, S. et al. Normalization of the Vasculature for Treatment of Cancer and Other Diseases. Physiol. Rev. 91, 1071-1121 (2011). 
19. Wilhelm, S. et al. Analysis of nanoparticle delivery to tumours. Nat. Rev. Mater. 1, 112 (2016).

20. Pepe, P. \& Aragona, F. Morbidity After Transperineal Prostate Biopsy in 3000 Patients Undergoing 12 vs 18 vs More Than 24 Needle Cores. Urology 81, 1142-1146 (2013).

21. Egbers, N., Schwenke, C., Maxeiner, A., Teichgraber, U. \& Franiel, T. MRI-guided core needle biopsy of the prostate: acceptance and side effects. Diagn. Interv. Radiol. 21, 215-221 (2015).

22. Shyamala, K., Girish, H.C. \& Murgod, S. Risk of tumor cell seeding through biopsy and aspiration cytology. J. Int. Soc. Prev. Community Dent. 4, 5-11 (2014).

23. Regev, A. et al. Sampling error and intraobserver variation in liver biopsy in patients with chronic HCV infection. Am. J. Gastroenterol. 97, 2614-2618 (2002).

24. Zhang, X.Y. et al. Study on pharmacokinetics and tissue distribution of single dose oral tryptanthrin in Kunming mice by validated reversed-phase high-performance liquid chromatography with ultraviolet detection. Interg. Med. Res. 6, 269-279 (2017).

25. Turfus, S.C., Delgoda, R., Picking, D. \& Gurley, B.J. Pharmacokinetics. Pharmacognosy: Fundamentals, Applications and Strategies Chapter 25, $495-512$ (2017).

26. Ernsting, M.J., Murakami, M., Roy, A. \& Li, S.-D. Factors controlling the pharmacokinetics, biodistribution and intratumoral penetration of nanoparticles. $J$. Control. Release 172, $782-794$ (2013).

27. Mage, P.L. et al. Closed-loop control of circulating drug levels in live animals. Nat. Biomed. Eng. 1, 1-10 (2017).

28. Li, H. et al. A Biomimetic Phosphatidylcholine-Terminated Monolayer Greatly Improves the In Vivo Performance of Electrochemical Aptamer-Based Sensors. Angew. Chem. Int. Ed. 56, $7492-7495$ (2017).

29. Li, H., Dauphin-Ducharme, P., Ortega, G. \& Plaxco, K.W. Calibration-Free Electrochemical Biosensors Supporting Accurate Molecular Measurements Directly in Undiluted Whole Blood. JACS 139, 11207-11213 (2017).

30. Asai, K. et al. An electrochemical aptamer-based sensor prepared by utilizing the strong interaction between a DNA aptamer and diamond. Analyst 145, 544-549 (2020).

31. Li, S.G. et al. A wrinkled structure of gold film greatly improves the signaling of electrochemical aptamer-based biosensors. RSC Adv. 11, 671-677 (2021).

32. Ferguson, B.S. et al. Real-Time, Aptamer-Based Tracking of Circulating Therapeutic Agents in Living Animals. Sci. Transl. Med. 5, 213 ra165 (2013).

33. Fu, K. et al. Accelerated electron transfer in nanostructured electrodes improves the sensitivity of electrochemical biosensors. bioRxiv, DOI: https://doi.org/10.1101/2021.1104.1113.439686 (2021).

34. Daggumati, P., Matharu, Z., Wang, L. \& Seker, E. Biofouling-Resilient Nanoporous Gold Electrodes for DNA Sensing. Anal. Chem. 87, 8618-8622 (2015).

35. Patel, J. et al. Electrochemical Properties of Nanostructured Porous Gold Electrodes in Biofouling Solutions. Anal. Chem. 85, 11610-11618 (2013).

36. Weltman, A., Yoo, J. \& Meng, E. Flexible, Penetrating Brain Probes Enabled by Advances in Polymer Microfabrication. Micromachines 7, 180 (2016).

37. Xie, C. et al. Three-dimensional macroporous nanoelectronic networks as minimally invasive brain probes. Nat. Mater. 14, 1286-1292 (2015).

38. Liu, J. et al. Syringe-injectable electronics. Nat. Nanotechnol. 10, 629-636 (2015).

39. Seo, J.-W. et al. Artifact-Free 2D Mapping of Neural Activity In Vivo through Transparent Gold Nanonetwork Array. Adv. Funct. Mater. 30, 2000896 (2020). 
40. Lai, R.Y., Plaxco, K.W. \& Heeger, A.J. Aptamer-Based Electrochemical Detection of Picomolar Platelet-Derived Growth Factor Directly in Blood Serum. Anal. Chem. 79, 229-233 (2007).

41. Xiao, Y., Lubin, A.A., Heeger, A.J. \& Plaxco, K.W. Label-Free Electronic Detection of Thrombin in Blood Serum by Using an Aptamer-Based Sensor. Angew. Chem. Int. Ed. 44, 5456-5459 (2005).

42. Lai, R.Y., Seferos, D.S., Heeger, A.J., Bazan, G.C. \& Plaxco, K.W. Comparison of the Signaling and Stability of Electrochemical DNA Sensors Fabricated from 6-or 11Carbon Self-Assembled Monolayers. Langmuir 22, 10796-10800 (2006).

43. Nakamura, K. et al. Characterization of mouse melanoma cell lines by their mortal malignancy using an experimental metastatic model. Life Sci. 70, 791-798 (2002).

44. Boucher, Y., Baxter, L.T. \& Jain, R.K. Interstitial Pressure-Gradients in TissueIsolated and Subcutaneous Tumors: Implications for Therapy. Cancer Res. 50, 44784484 (1990).

45. Grantab, R., Sivananthan, S. \& Tannock, I.F. The Penetration of Anticancer Drugs through Tumor Tissue as a Function of Cellular Adhesion and Packing Density of Tumor Cells. Cancer Res. 66, 1033-1039 (2006).

46. Provenzano, P.P. et al. Enzymatic Targeting of the Stroma Ablates Physical Barriers to Treatment of Pancreatic Ductal Adenocarcinoma. Cancer Cell 21, 418-429 (2012).

47. Idili, A. et al. Seconds-resolved pharmacokinetic measurements of the chemotherapeutic irinotecan in situ in the living body. Chem. Sci. 10, 8164-8170 (2019).

48. Clarke, W. \& Marzinke, M.A. Basic pharmacokinetics. Contemporary Practice in Clinical Chemistry Chapter 50, 895-904 (2020).

49. Heldin, C.-H., Rubin, K., Pietras, K. \& Ostman, A. High interstitial fluid pressure - An obstacle in cancer therapy. Nat. Rev. Cancer 4, 806-813 (2004).

50. Yonucu, S., Yilmaz, D., Phipps, C., Unlu, M.B. \& Kohandel, M. Quantifying the effects of antiangiogenic and chemotherapy drug combinations on drug delivery and treatment efficacy. Plos Comput. Biol. 13, 1-17 (2017).

51. Torchilin, V. Tumor delivery of macromolecular drugs based on the EPR effect. Adv. Drug Deliver. Rev. 63, 131-135 (2011). 